\title{
Evaluación y rediseño de sistemas de abastecimiento de agua potable
}

Evaluation and redesign of drinking water supply networks

Yeferson Sergio Romero Nina

sergioromeronina@gmail.com

Código ORCID: 0000-0002-8239-3498
4 Articulo aceptado en septiembre 2020

< Arbitrado en octubre 2020

< Publicado en enero 2021

\section{Universidad Autónoma Tomás Frías, Potosí - Bolivia}

Resumen

En Bolivia existen comunidades menores que viven en condiciones insalubres por falta de agua potable, en este sentido el presente estudio se propone evaluar y optimizar el sistema de abastecimiento de agua potable de la comunidad Caiza D, ubicada en Potosí. Con un enfoque mixto de corte longitudinal se aplica un proceso validado por criterio de expertos, que consiste en calibrar y llevar redes en funcionamiento a un software libre que provee información para la toma de decisiones ingenieriles. Se determina que la bomba deberá tener 1,9 $\mathrm{Hp}$ de potencia, cuatro (4) pulgadas de diámetro y capacidad de 6,4 l/s. Además, el tanque considerará un consumo promedio anual de 1,5E+05 y un volumen del reservorio de $25 \%$ de $Q m, V=37 m 3$. El rediseño del sistema implicará renovar bomba de agua y tanque de almacenamiento y se considera factible usar la red de agua existente, previo mantenimiento y renovaciones de componentes oxidados.

Palabras clave: Red de agua potable; calibración hidráulica; modelo hidráulico; aducción

\section{Abstract}

In Bolivia there are smaller communities that live in unsanitary conditions due to lack of drinking water. In this sense, the present study set out to evaluate and later optimize the drinking water supply system of Caiza D community, located in Potosí. With a mixed approach of longitudinal cutting, a process validated by expert criteria was applied, which consists of calibrating and bringing networks in operation to free software that provides information for engineering decisions. It was determined that the pump should have $1.9 \mathrm{HP}$ of power, 4 inches in diameter and a capacity of $6,4 \mathrm{l} / \mathrm{s}$, the tank will consider an average annual consumption of $1.5 \mathrm{E}+05$ and a reservoir volume of $25 \%$ of $\mathrm{Qm}, \mathrm{V}=$ $37 \mathrm{~m} 3$. The redesign of the system will involve renovating the water pump and storage tank and it was considered feasible to use the existing water network, after maintenance and renovations of rusted components.

Keywords: Drinking water network; hydraulic calibration; hydraulic model; adduction 
INTRODUCCIÓN

Los sistemas de abastecimientos de agua potable permiten el aprovisionamiento de agua a las ciudades y un mal funcionamiento impacta negativamente en toda la población. En los países en desarrollo aún existen localidades que no cuentan con este suministro a cabalidad. Este es el caso de Bolivia, donde existen poblaciones en las que el sistema de agua potable presenta deficiencias y no abastece al 100\%, produciendo problemas relacionados con la calidad de vida, salud y alimentación.

El problema se manifiesta principalmente en comunidades con población reducida, como en la comunidad de Caiza D (ubicada al sud este de la ciudad de Potosí), que cuenta con una población cercana a 1000 habitantes. En la comunidad existe un sistema de abastecimiento cuya fuente permitía el abastecimiento de la demanda de la población. Actualmente, su cobertura ha disminuido $y$, según la norma boliviana que regula el abastecimiento de agua, está muy por debajo del flujo aceptable (Reglamento Nacional, 2004). Las causas son diversas: uso del agua para el riego de las parcelas aledañas a la tubería de aducción, necesidad de mantenimiento y crecimiento de la población.

Se sabe que el servicio de agua potable podría ser mejorado incrementando el caudal para que el aprovisionamiento sea regular. En este sentido, es importante determinar el funcionamiento de la red en términos hidráulicos y así determinar la posibilidad de utilizar la infraestructura existente o confirmar la necesidad de renovación. Se utiliza entonces la calibración como mecanismo que permite averiguar la fiabilidad del modelo hidráulico y la veracidad de los datos utilizados para simular los sistemas (AWWA, 2012).

El presente estudio tuvo por objetivo evaluar el sistema de abastecimiento de agua potable de la comunidad Caiza D, mediante la modelación del sistema hidráulico. Este propósito tiene relevancia social, no sólo para la comunidad Caiza D, sino para todas las comunidades dispersas en Bolivia que aún no tienen acceso suficiente de agua potable. El proceso de evaluación propuesto puede replicarse y evaluar así otras redes de agua.

El estudio se justifica en la medida en que sea difundido y considerado por la comunidad de ingenieros y tomadores de decisiones sobre temas de agua. Es fundamental comprender que las redes de abastecimiento de agua segura, adecuada y accesible, juntamente con un saneamiento apropiado, son, sin duda, necesidades básicas y componentes esenciales de la atención primaria de la salud.

En el presente estudio se presenta la base teórica del modelo hidráulico aplicado. El propósito de este modelo está relacionado con el análisis hidráulico que se necesita y puede ser simulación en régimen estacionario $o$ simulación en período extendido (Ostfeld, 2012; Walski, 2003). Se 
identifican cinco (5) aplicaciones para las cuales es posible construir un modelo: planes maestros, estudios de protección contra incendios, estudios de calidad del agua, estudios de eficiencia energética $y$, finalmente, operación de la red (Christie et al, 2011). Sin embargo, los estudios de calidad de agua exigen un mayor nivel de calibración (Larado, 2012).

La calibración de modelos hidráulicos de redes de distribución es un proceso lógico sencillo, que involucra determinar aquello que está mal en el modelo y corregir los errores para lograr que el modelo funcione adecuadamente. Resulta improbable que el problema de calibración de modelos hidráulicos sea resuelto utilizando un procedimiento analítico simple o una técnica de optimización. Sin embargo, existen consideraciones generales que pueden ayudar a su aproximación (Ormsbee, 1994). Se considera el siguiente procedimiento general de siete (7) pasos al momento de abordar un proceso de calibración (Hutton, 2014): (a) identificar el propósito del modelo, (b) determinar el valor inicial de los parámetros a estimar, (c) recolectar datos de calibración, (d) evaluar los resultados del modelo, (e) calibrar a nivel macro, (f) realizar un estudio de sensibilidad $y$, finalmente, (g) calibrar a nivel micro.

Por otra parte, según Shamir (1977), la calibración consiste en determinar las características físicas y operacionales de un sistema existente, aplicando un modelo computacional que permita obtener resultados realistas. Walski (2003) la define como un proceso de dos (2) pasos que consisten en: (a) Comparar las presiones y caudales simulados con aquellos observados (medidos) para unas condiciones de operación conocidas y (b) Ajustar los datos de entrada al modelo de tal forma que exista concordancia entre los valores simulados y observados.

La calibración es una garantía para la aplicabilidad del modelo y su importancia sustenta en tres (3) aspectos básicos (Walski, 2003): confianza, conocimiento y compresión y resolución de problemas. Ésta termina cuando se alcanza la tolerancia esperada $10 \%$ de diferencia entre los valores medidos y simulados según los estándares normalmente aceptados).

Los datos de calibración pueden ser recolectados mediante: (a) pruebas de campo realizadas en hidrantes para obtener datos de caudal y presión (Lansey, 2000), (b) Datos de telemetría (SCADA) correspondientes a series de tiempo para caudales, presiones, y niveles de tanques (Walski, 2003) $y$, finalmente, (c) Datos referentes a pruebas con trazadores químicos conservativos 0 no conservativos (Ormsbee, 1994).

Evaluar los resultados de una simulación permite medir la presión de un modelo hidráulico, comparando los resultados y las mediciones realizadas en campo (Lansey, 2000). La calidad de los datos recabados será determinante 
para el éxito de la modelación, Walski (2003) realiza una clasificación cualitativa de la calidad de los datos, y propone tres (3) tipos: buenos (good data), malos (bad data) e inútiles (useless data).

Existen errores que provocan discrepancias entre valores simulados y observados, identificarlos y minimizarlos requiere de la aplicación iterativa de tres (3) procesos: macro-calibración, micro-calibración y análisis de identificar aquellos parámetros que generan mayor impacto en los resultados de la simulación.

\section{MATERIALES Y MÉTODOS}

Se trata de una investigación aplicada de corte longitudinal que, mediante la modelación de un sistema hidráulico, pudo evaluar la calidad de un sistema de redes de abastecimiento de sensibilidad. La macro-calibración se enfoca en la calibración de toda la red de distribución o zonas de presión y consiste en identificar y corregir las fuentes de error que ocasionan las diferencias más significativas (Molina, 2015). El análisis de sensibilidad consiste en variar los parámetros del modelo, cuantificar el efecto sobre los resultados de la simulación asociado a cada una de estas variaciones e

agua potable y, posteriormente, gracias a la simulación, presenta el esquema del rediseño de la misma red. El proceso que se aplica para evaluar y rediseñar el sistema de abastecimiento de agua potable de la comunidad Caiza D combina los lineamientos propuestos por Walski (2013) y Hulton (2014), según se muestra en la tabla 1.

Tabla 1. Procesos para la calibración de redes de agua

\begin{tabular}{|c|c|c|}
\hline Etapas según Hutton (2014) & Etapas según Walski (2003) & Etapas del proceso aplicado \\
\hline Identificar el propósito del modelo & & $\begin{array}{l}\text { Reconocimiento y diagnóstico del } \\
\text { funcionamiento del sistema. }\end{array}$ \\
\hline $\begin{array}{l}\text { Determinar el valor inicial de los } \\
\text { parámetros a estimar }\end{array}$ & & $\begin{array}{l}\text { Recopilación de métricas en campo } \\
\text { (presiones y caudales) que permitieron } \\
\text { aplicar los modelos de simulación. }\end{array}$ \\
\hline Recolectar datos de calibración & & \\
\hline Evaluar los resultados del modelo & & Rediseño y calibración la red de agua. \\
\hline $\begin{array}{l}\text { Realizar una calibración a nivel } \\
\text { macro }\end{array}$ & $\begin{array}{l}\text { Comparar las presiones y caudales } \\
\text { simulados con aquellos observados }\end{array}$ & \\
\hline $\begin{array}{l}\text { Realizar un análisis de sensibilidad } \\
\text { Realizar una calibración a nivel } \\
\text { micro }\end{array}$ & $\begin{array}{l}\text { Ajustar los datos de entrada al } \\
\text { modelo }\end{array}$ & $\begin{array}{l}\text { Desarrollo modelo hidráulico que } \\
\text { optimiza el funcionamiento de la red }\end{array}$ \\
\hline
\end{tabular}


En la tercera columna de la tabla 1 se muestra el proceso propuesto, comprende cinco (5) etapas. Este tiene un enfoque mixto, ya que comprende variables cuantitativas $y$ cualitativas. Este fue validado aplicando la técnica del juicio de expertos, la consulta se hizo al Colegio de Ingenieros de Bolivia, quienes recomendaron a profesionales con amplia trayectoria y conocimiento en redes de abastecimiento de agua potable.

Con este fin los paquetes informáticos empleados para procesar la información fueron AutoCADß y AutoCAD Civil 3D®. Asimismo se aplicó el paquete informático para modelación de redes de agua potable: Epanet $\mathrm{v} 2.0 \AA$ y la extensión Epacad® que permite la modelación de la red. Para el manejo de imágenes satelitales se utiliza el Google Maps.

Para las mediciones de parámetros hidráulicos en campo se cuenta con un caudalímetro $8188-29$ Original System (Gardena), con las siguientes especificaciones técnicas: (a) Permite mediciones de caudal en tuberías a presión de entre 1" y 360" y (b) la precisión de la medición es de $\pm 0.5 \%$, para líquidos homogéneos. También se cuenta con un presurímetro marca y modelo MARSH WV 26050-0607, con un rango de medición: 0-160 psi, precisión $\pm 0.25 \%$ y con temperatura de funcionamiento de -20 a $70{ }^{\circ} \mathrm{C}$.

$\mathrm{Se}$ realiza el levantamiento topográfico para modificar la aducción y la zona de la Red (referenciado a bancos de Nivel "Bench Nark"). Las nivelaciones en las poligonales fueron cada $5 \mathrm{~m}$ y también se considera terrenos con pendiente abrupta. Por su parte, las mediciones se efectuaron en cámaras ingreso a domicilio cuya tubería es de PVC. Para el dimensionamiento de la red de distribución y conducciones en general se aplica la fórmula de William \& Hazen con la ecuación (1).

$$
J=10.64605 * Q^{\wedge} 1.852 \div C^{\wedge} 1.852 \div D^{\wedge} 4.8704
$$

\section{Donde:}

$\mathrm{J}$ : pérdida de carga unitaria $(\mathrm{m} / \mathrm{m})$

Q: es el caudal $\left(\mathrm{m}^{3} / \mathrm{s}\right)$

C: coeficiente de rugosidad, que depende del material (adimensional $100-140$ )

D: diámetro de la tubería (plg).

\section{RESULTADOS}

$\mathrm{Se}$ presenta el proceso de calibración de la red de agua potable para la comunidad Caiza $D$, según el proceso ya especificado de cinco etapas.

\section{Reconocimiento y diagnóstico del funcionamiento del sistema}

El reconocimiento considera las condiciones actuales con relación a: obra de toma, aducción, tanque de almacenamiento, red de abastecimiento y proyección de crecimiento demográfico. Se presenta la síntesis del diagnóstico en la Tabla 2. 
Las mediciones de parámetros hidráulicos en campo son indispensables para calibrar un sistema hidráulico y, para este fin, es importante también la contemporaneidad de las mediciones. Se lleva a cabo para tal efecto las mediciones periódicas de parámetros hidráulicos en la red. Las mediciones se obtienen en diferentes periodos de tiempo, comprendidos entre 2015 y 2016. Los parámetros hidráulicos que se midieron son los siguientes: caudal en diferentes puntos estratégicos de la red actual y presión en los mismos puntos donde se midieron los caudales.

Tabla 2. Síntesis del diagnóstico de la comunidad (sin proyecto)

\begin{tabular}{|c|c|}
\hline COMPONENTE & DESCRIPCIÓN \\
\hline OBRA DE TOMA & $\begin{array}{l}\text { - } \quad \text { No se cuenta con cámara de llaves. } \\
\text { - } \text { las familias y para riego. Abastece alrededor del } 70 \% \text { del consumo } \\
\text { básico familiar, existe escases. } \\
\text { - Se percibe que no ha habido mantenimiento en los últimos años. } \\
\text { - La cámara colectora requiere reparación o renovación. }\end{array}$ \\
\hline
\end{tabular}

ADUCCIÓN

TANQUE DE

ALMACENAMIENTO

RED DE

ABASTECIMIENTO

PROYECCIONES DEMOGRÁFICAS
- La aducción se encuentra muy deteriorada.

- Filtración importante

- Se requiere nueva aducción.

- No posee tapa sanitaria.

- El caudal que recibe es casi la mitad del que se aforó en la obra de toma.

- El tanque se encuentra en mal estado, debido a la acumulación de arena y falta de mantenimiento.

- Presenta conexiones en mal estado.

- Se verifica que las tuberías son antiguas lo que hace que haya demasiada fricción en sus paredes interiores.

- Tuberías inadecuadas para trabajar a presión.

- La operación de la red se realiza por personal no autorizado.

- Las presiones de campo con las que opera el sistema son muy bajas, no permite la operación de aparatos sanitarios.

- Es necesario darle una combinación de diámetros adecuada para así cumplir con los requerimientos según Norma Boliviana NB689.

- Según datos del censo nacional la población tiene tasa crecimiento positiva, el cálculo de la proyección de crecimiento se realizó con base en los dos últimos censos nacionales (1992 y 2001) y al último censo comunitario (2011). 
Las mediciones se efectuaron en cámaras ingreso a domicilio cuya tubería es de PVC. Se obtuvieron los siguientes datos de campo promediados en cuanto a presiones y caudales, asumiendo un modelo estacionario en fechas de estiaje.

Una vez completados los pasos precedentes, se procede a iniciar las corridas de simulación confirmando antes los datos insertados (validación) y después fijando los parámetros operativos: unidades de caudal LPS, fórmula de pérdidas $\mathrm{H}-\mathrm{W}$, peso específico relativo 1 , viscosidad relativa 1, máximo iteraciones 60, precisión 0,01, continuar en caso número equilibrio, curva modulaciones 1, factor de demanda 1.

\section{Recopilación de métricas en} campo

Se usa la calibración como mecanismo que para averiguar la fiabilidad del modelo y la veracidad de los datos utilizados para simular los sistemas. La Figura 1 permite visualizar el esquema de nudos y tuberías de la red, según el reporte de medición la red actual cuenta con 190 tuberías con longitud total de $2.512,98 \mathrm{~m}$, con diámetros entre 20,80 y $66,80 \mathrm{~mm}$.

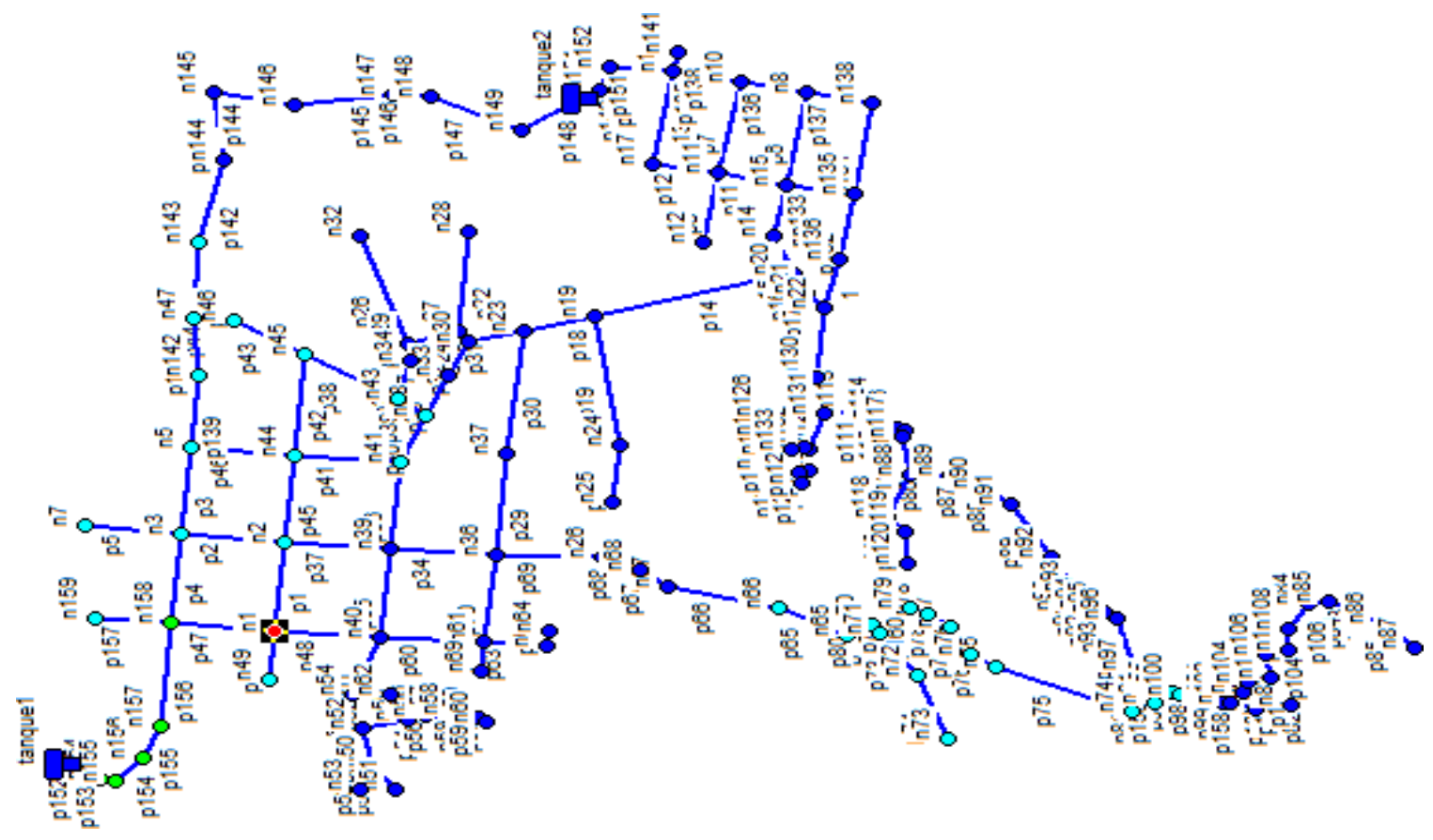

Figura 1. Esquema de nudos y tuberías

En la tabla 3 se muestran los estadísticos finales en cuanto a caudales y presiones. 
Tabla 3. Resumen Estadístico Prueba T de Student para la Presiones y Caudales

\begin{tabular}{lcccc}
\hline \multicolumn{1}{c}{ Estadístico } & \multicolumn{2}{c}{ PRESIONES } & \multicolumn{2}{c}{ CAUDALES } \\
& Var 1 & Var 2 & Var 1 & Var 2 \\
\hline Media & 8,74 & 9,25 & 1,06 & 0,97 \\
Varianza & 7,67 & 8,65 & 1,09 & 1,56 \\
Observaciones & 14 & 14 & 9 & 9 \\
Coeficiente de correlación de Pearson & 0,979 & & 0,982 & \\
Diferencia hipotética de las medias & 0 & & 0 & \\
Grados de libertad & 13 & & 8 & \\
Estadístico t & $-3,147$ & & 0,844 & \\
$\mathrm{P}(\mathrm{T}<=\mathrm{t})$ una cola & 0,004 & & 0,212 & \\
Valor crítico de t (una cola) & 1,771 & & 1,860 & \\
$\mathrm{P}(\mathrm{T}<=\mathrm{t}$ ) dos colas & 0,008 & & 0,423 & \\
Valor crítico de t (dos colas) & $\underline{2,160}$ & & $\underline{2,306}$ & \\
\hline
\end{tabular}

El proceso de calibrar implica varias corridas hasta alcanzar a reflejar el sistema actual (debido a inconsistencias en la topología de la red). Se logra en la mayoría de los puntos una diferencia entre lo medido y lo simulado del $10 \%$. El reporte estadístico presentado en la tabla 2 indica que a través de las pruebas y medidas tomadas tanto en campo como en el programa el coeficiente de correlación es superior a 0.9 , lo que significa que las muestras son estadísticamente similares.
Luego de presentar las características técnicas de los diferentes elementos que componen el sistema de abastecimiento de la red de agua potable y con todos los datos obtenidos en campo y en simulación, se procedió a identificar las alternativas seleccionadas a las principales problemáticas del funcionamiento actual del sistema. Con este fin se estima la proyección de la demanda, según se presenta en la Figura 2. 


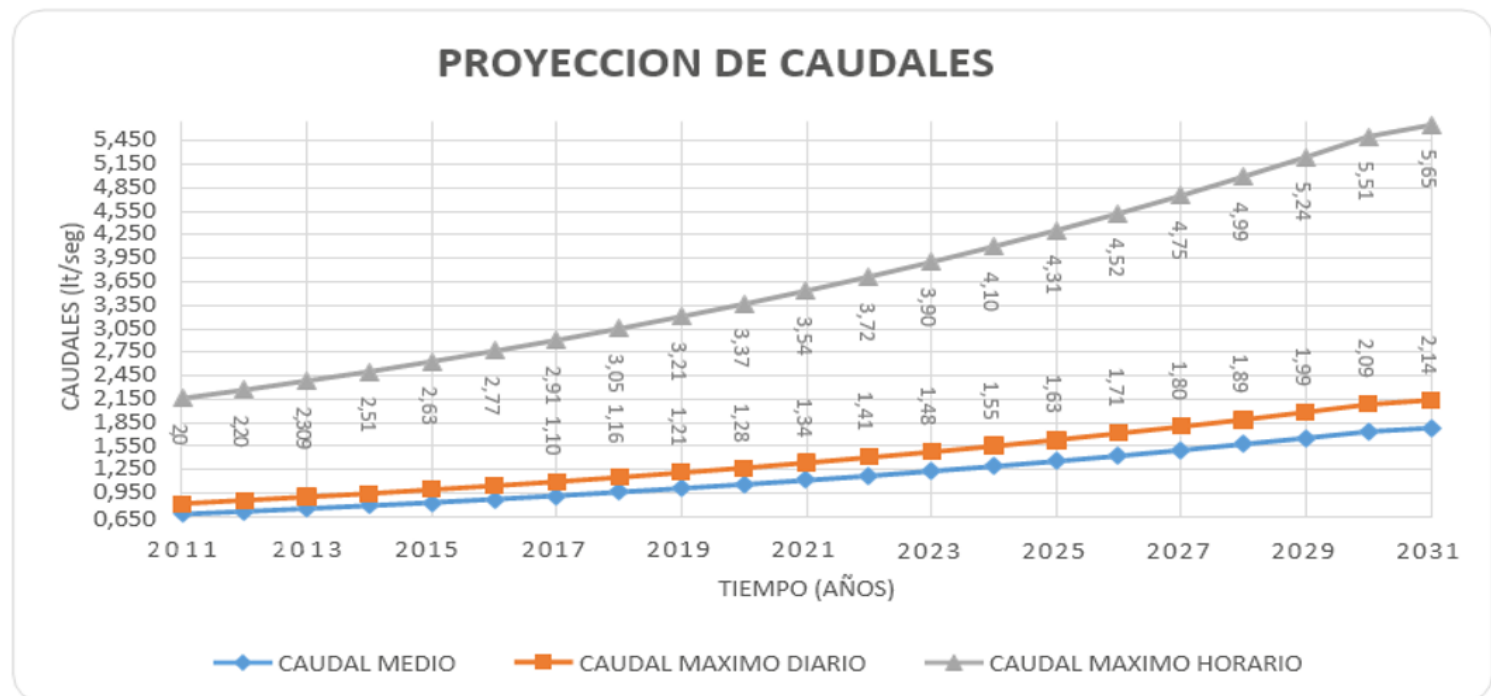

Figura 2. Proyección de la demanda

Según la proyección de la demanda presentada en la Figura 2, se determina un caudal medio diario de Qmd de 1,785 [l/s], causal máximo diario de Qmaxd de 2,142 [l/s] y un caudal máximo horario de Qmáxh de 5.654 [l/s].

Por una comparación de los resultados obtenidos del análisis de aguas realizado para la fuente del sistema en estudio con los valores referenciales de la norma, la calidad del agua es apta para consumo, en cuanto a sus parámetros físicos, químicos y bacteriológicos.

Se compara los resultados del análisis del agua con los valores referenciales de la NB-689. El análisis físico determinó: (a) turbiedad de 0,35, muy por debajo del valor máximo aceptable que es 5 , (b) Color 5 UC en escala Pt-Co, 1/3 del máximo aceptable que es 15 UC, (c) $365 \mathrm{mg} / \mathrm{l}$ sólidos totales disueltos, siendo 1000 mg/l el máximo aceptable y, finalmente, (d) Las características de olor, sabor y temperatura están dentro de los parámetros aceptables. Según el análisis químico las características del agua se encuentran dentro de los márgenes aceptables para el consumo humano, se considera las variables: dureza, calcio, magnesio, manganeso, hierro, sulfato, cloruro, fluoruros, nitratos, nitritos y $\mathrm{pH}$. Por su parte, el análisis bacteriológico dio negativo en coliformes totales y en Escherichia coli (E. coli). Finalmente, se realizaron análisis complementarios que determinaron inexistencia de Demanda Bioquímica de Oxígeno $\mathrm{DBO}_{5}$ y Oxígeno disuelto OD y conductividad de $606 \mathrm{mmhos} / \mathrm{cm}$, siendo la máxima aceptada 1500 $\mathrm{mmhos} / \mathrm{cm}$.

\section{Rediseño y calibración la red de agua}

Dada la identificación del estado actual del sistema de abastecimiento de agua potable en la comunidad de Caiza $D$ en flujo estacionario, y encontrando deficiencias en su funcionamiento es preciso identificar los 
parámetros a considerar para el planeamiento de la optimización hidráulica de la red: demanda y presiones de servicio.

Realizadas las estimaciones se determinaron los valores requeridos para el diseño de la bomba de agua: caudal del bombeo de 6,4 l/s, diámetro mínimo de 4 plg, pérdidas de carga desde la aducción al punto de bomba (Hazen Williams) de 1,796 $\mathrm{m}$, pérdidas de carga de la bomba al tanque de $0,0026 \mathrm{~m} / \mathrm{m}$, carga total de $46,5 \mathrm{~m}$ y cálculo de la potencia de la bomba de $1.9 \mathrm{Hp}$. Por tanto, según los criterios de selección del tipo de bomba que se determina tiene 2 $\mathrm{Hp}$ de potencia y con una capacidad de $10 \quad \mathrm{l} / \mathrm{s}$. Garantizando el funcionamiento de la aducción. Se usará la bomba 40-315.

El cálculo de la demanda de la red actual sive para evaluar y calibrar la red y generar presiones óptimas de servicio, considerando los lineamientos dados por los Reglamentos del Agua y la Norma NB 689. Se traza una red matriz compuesta por una serie de diámetros comerciales a fin de optimizar su funcionamiento, permitiendo proyectar un nuevo diseño de la aducción que incluye una bomba que permitirá aprovechar entre un 80 y $100 \%$ la obra de toma.

Considerando que la bomba se posicionó en la progresiva $0+683,68$, se realiza el cálculo de la nueva línea aducción. Para este cálculo se utiliza la ecuación de Hazen - Williams, en cuanto a la obtención de pérdidas por fricción se utiliza un coeficiente $C$ de 150 para un material de PVC. En tal caso se determinaron los valores de pérdida de carga, con sus respectivas cotas piezométricas, según se presenta en la tabla 4. 
Tabla 4. Reporte de la nueva aducción

\begin{tabular}{|c|c|c|c|c|c|c|c|c|c|c|}
\hline $\begin{array}{l}\text { :0 } \\
\stackrel{0}{20}\end{array}$ & 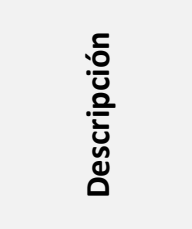 & $\overline{\underline{\xi}}$ & $\frac{\bar{\xi}}{\frac{\bar{d}}{\Psi}}$ & $\begin{array}{l}\text { घo } \\
\stackrel{0}{0} \\
\bar{\sigma}\end{array}$ & $\stackrel{+}{\pi}^{\frac{\pi}{2}}$ & 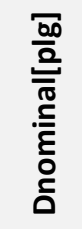 & 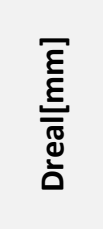 & $\frac{\bar{\xi}}{\underline{\xi}}$ & $\underset{\underline{\Sigma}}{\underline{\underline{\xi}}}$ & 鹃 \\
\hline 0,00 & $C A$ & 0,00 & 3172,209 & 2,1417819 & PVC & 4,00 & 105,1 & $6,32 \mathrm{E}-04$ & $0,00 E+00$ & 3172,21 \\
\hline 158,70 & Tubería p1 & 158,70 & 3170,559 & 2,1417819 & PVC & 3,00 & 81,7 & $8,84 \mathrm{E}-04$ & $1,40 \mathrm{E}-01$ & 3170,56 \\
\hline 372,20 & Tubería p10 & 9,67 & 3167,97 & 2,1417819 & PVC & 3,00 & 81,7 & $8,84 \mathrm{E}-04$ & $8,55 \mathrm{E}-03$ & 3167,97 \\
\hline 461,69 & Tubería p11 & 89,49 & 3167,381 & 2,1417819 & PVC & 3,00 & 81,7 & $8,84 \mathrm{E}-04$ & $7,91 \mathrm{E}-02$ & 3167,38 \\
\hline 501,80 & Tubería p12 & 40,11 & 3165,353 & 2,1417819 & PVC & 3,00 & 81,7 & $8,84 \mathrm{E}-04$ & $3,55 \mathrm{E}-02$ & 3165,35 \\
\hline 584,76 & Tubería p13 & 82,96 & 3166,395 & 2,1417819 & PVC & 3,00 & 81,7 & $8,84 \mathrm{E}-04$ & $7,34 \mathrm{E}-02$ & 3166,39 \\
\hline 586,71 & Tubería p15 & 1,95 & 3165,32 & 2,1417819 & PVC & 3,00 & 81,7 & 8,84E-04 & $1,73 \mathrm{E}-03$ & 3165,32 \\
\hline 596,48 & Tubería p16 & 9,77 & 3165,541 & 2,1417819 & PVC & 3,00 & 81,7 & $8,84 \mathrm{E}-04$ & $8,63 \mathrm{E}-03$ & 3165,54 \\
\hline 600,58 & Tubería p17 & 4,10 & 3165,528 & 2,1417819 & PVC & 3,00 & 81,7 & $8,84 \mathrm{E}-04$ & $3,62 \mathrm{E}-03$ & 3165,53 \\
\hline 606,88 & Tubería p18 & 6,30 & 3166,014 & 2,1417819 & PVC & 3,00 & 81,7 & 8,84E-04 & 5,57E-03 & 3166,01 \\
\hline 609,33 & Tubería p19 & 2,45 & 3166,251 & 2,1417819 & PVC & 3,00 & 81,7 & 8,84E-04 & 2,17E-03 & 3166,25 \\
\hline 641,88 & Tubería p26 & 2,75 & 3164,668 & 2,1417819 & PVC & 3,00 & 81,7 & $8,84 \mathrm{E}-04$ & $2,43 \mathrm{E}-03$ & 3164,67 \\
\hline 657,53 & Tubería p30 & 2,32 & 3164,26 & 2,1417819 & PVC & 3,00 & 81,7 & $8,84 \mathrm{E}-04$ & $2,05 \mathrm{E}-03$ & 3164,26 \\
\hline 666,99 & Tubería p31 & 9,46 & 3164,422 & 2,1417819 & PVC & 3,00 & 81,7 & 8,84E-04 & $8,36 \mathrm{E}-03$ & 3164,42 \\
\hline 683,68 & BOMBA & 16,69 & 3164,171 & 2,1417819 & PVC & 3,00 & 81,7 & $8,84 \mathrm{E}-04$ & 1,48E-02 & 3164,17 \\
\hline 697,60 & ría p33 & 13,92 & 3165,159 & 2,1417819 & PVC & 3,00 & 81,7 & 3,84E-04 & $1,23 \mathrm{E}-02$ & 3165,16 \\
\hline
\end{tabular}

En la tabla 4 se presenta el reporte de la nueva aducción, el cual permite verificar que las tuberías en la red están funcionando dentro de los parámetros requeridos de pérdida de fricción y cota piezométrica. La cota piezométrica de la bomba de agua es $3.164,17 \mathrm{~m}$.

Realizadas las estimaciones se determina los valores requeridos para el tanque de almacenamiento: consumo promedio anual, $Q m$ de 1,5E+05 I, volumen del reservorio considerando
$25 \%$ de $Q m$ de $37 m^{3}$, con el valor del volumen (V) se define en reservorio de sección cuadrada que se obtiene de la Norma: PL-AP-13-05 Tanque Elevado De $\mathrm{H}^{\circ} \mathrm{A}^{\circ}$ PARA $50 \mathrm{M3}$. Por tanto, se determina que el tanque de almacenamiento será del tipo elevado, diseñado en hormigón armado con una altura total de 20 m, según NB-689.

Desarrollo modelo hidráulico que optimiza el funcionamiento de la red 
Se determina para cada tramo de todo el circuito las pérdidas de carga correspondientes y las razones de estas al caudal considerado en cada caso la fórmula de Hazen- Williams. Se realizan verificaciones en el programa Epanet®, en cuanto a las tuberías de la red permite tomar criterios para determinar los diámetros de redes considerando presiones y velocidades. La tabla 5 es representativa de los resultados obtenidos.

Tabla 5. Reporte de la red propuesta

\begin{tabular}{cccccccc}
\hline ID Línea & $\begin{array}{c}\text { Longitud } \\
\mathbf{m}\end{array}$ & $\begin{array}{c}\text { Diámetro } \\
\text { plg }\end{array}$ & Rugosidad & $\begin{array}{c}\text { Caudal } \\
\text { LPS }\end{array}$ & $\begin{array}{c}\text { Velocidad } \\
\mathbf{m} / \mathbf{s}\end{array}$ & $\begin{array}{c}\text { Pérdida Unit. } \\
\mathbf{m} / \mathbf{k m}\end{array}$ & Factor Fricción \\
\hline Tubería p1 & 74,56 & 0,75 & 140 & 0,05 & 0,3 & 1,8 & 0,036 \\
Tubería p2 & 71,96 & 1,5 & 140 & $-2,05$ & 1,58 & 70,48 & 0,023 \\
Tubería p3 & 71,89 & 3 & 140 & 4,68 & 1,33 & 29,02 & 0,021 \\
Tubería p4 & 73,61 & 3 & 140 & 6,93 & 1,98 & 60,08 & 0,02 \\
Tubería p10 & 43,28 & 1 & 140 & $-0,29$ & 0,52 & 14,84 & 0,029 \\
Tubería p11 & 47,25 & 1 & 140 & $-0,37$ & 0,66 & 22,94 & 0,028 \\
Tubería p24 & 11,47 & 1 & 140 & 0,16 & 0,3 & 5,09 & 0,031 \\
Tubería p25 & 14,07 & 1 & 140 & 0,43 & 0,77 & 30,91 & 0,027 \\
Tubería p31 & 39 & 1 & 140 & $-0,6$ & 1,08 & 57,14 & 0,026 \\
Tubería p32 & 30,29 & 1 & 140 & $-0,45$ & 0,81 & 33,84 & 0,027 \\
\hline
\end{tabular}


En la tabla 5, se muestra una tabla representativa de todo el universo, se advierte que la pérdida unitaria y la velocidad se encuentran dentro del rango de parámetros admisibles.

Para la elaboración del presupuesto de inversión del proyecto se consideró el desglose de materiales, mano de obra y equipo para cada uno de los ítems de ejecución de la obra.

El tiempo requerido para la ejecución de la obra está planificado para ocho (8) meses y deberá estimarse el cronograma y moneda, ubicación de la obra, vías de acceso a la obra, disponibilidad de agua, disponibilidad de combustible y energía, condiciones climáticas, disponibilidad de mano de obra de la región, disponibilidad de materiales de construcción $y$, finalmente, bancos de préstamo y costo de transporte. Asimismo, se considera los porcentajes de herramientas menores, cargas sociales, gastos generales y administrativos impuestos y utilidades, estos están normados bajo la ley de contrataciones a través del Decreto Supremo Nro. 27328.

\section{CONCLUSIONES}

La investigación presenta el proceso de calibración en sistemas de abastecimiento de agua en la comunidad de Caiza D, proponiendo un proceso para obtener la calibración manual de la red. La novedad científica de este aporte radica en comparar los resultados simulados del modelo hidráulico con las mediciones obtenidas en campo, mediante varios ajustes al modelo, hasta alcanzar la concordancia entre los datos históricos obtenidos y la simulación. Una vez realizada la evaluación se determinó necesario el rediseñó del sistema de abastecimiento del suministro.

Para realizar el proceso de calibración es preciso disponer de información que permita caracterizar la red; visitas, aforos, censo poblacional. Asimismo, el trabajo de campo fue fundamental ya permitió conocer la magnitud real del proyecto. Se resalta la utilidad de las herramientas aplicadas, se sugiere el uso del Caudalímetro 818829 Original System (Gardena) Alemania, ya que permitió evaluar mediciones de caudales con un error de $\pm 0.5 \%$ y el Presurímetro MARSH WV 26050-0607 que permite hacer un control de presiones con un error de $\pm 0.25 \%$.

El procedimiento aplicado permite establecer la relación entre los sistemas de red de agua potable en ejecución y los diseños de sistemas de este suministro, ya que es una primera aproximación a la evaluación y calibración de redes de agua potable por demás importante para el futuro desarrollo socio económico de la localidad de Caiza D y otras comunidades pequeñas que aún no tienen un sistema de red de agua aceptable en Bolivia. En este sentido, se logró proponer un esquema de calibración hidráulica para el sistema de distribución de agua potable desarrollado con el software EPANETß, mediante la modelación hidráulica de 
la red evaluando el escenario actual. Asimismo, se realizó un análisis estadístico de las presiones de servicio, encontrando que es muy útil para la calibración de la red. Con este análisis se prueba que el escenario de calibración propuesto proporciona a la red las presiones adecuadas que se ajustan a la necesidad actual de la comunidad y principalmente de los usuarios.

\section{REFERENCIAS}

AWWA. American Water Works Association (Ed.). (2012). Manual de entrenamiento para operadores de sistemas de distribución de agua. American Water Works Association

Hutton, C. J., Kapelan, Z., VamvakeridouLyroudia, L., \& Savić, D. (2014). Application of formal and informal Bayesian methods for water distribution hydraulic model calibration. Journal of Water Resources Planning and Management, 140(1 1), 04014030

Lansey, K. E., \& Mays, L. W. (2000). Optimal design of water distribution systems. Water Distribution System Handbook, McGraw-Hill, New York Molina Arce, S. X. (2015). Metodología para la calibración preliminar de modelos de redes de distribución de agua mediante la utilización conjunta de demandas y consumos dependientes de la presión

Ormsbee, L. E., \& Lansey, K. E. (1994). Optimal control of water supply pumping systems. Journal of Water Resources Planning and Management, 120(2), 237-252. Ostfeld, A., Salomons, E., Ormsbee, L., Uber, J. G., Bros, C. M., Kalungi, P., ... \& McKillop, R. (2012). Battle of the water calibration networks. Journal of Water Resources Planning and Management, 138(5), 523-532

Reglamento Nacional NB 689. (2004). REGLAMENTOS TÉCNICOS DE DISEÑO PARA SISTEMAS DE AGUA POTABLE. Ministerio de Agua Viceministerio de servicios básicos. Bolivia

Shamir, U., \& Howard, C. D. (1977). Engineering analysis of waterdistribution systems. Journal (American Water Works Association), 510-514.

Walski, T. M., Chase, D. V., Savic, D. A., Grayman, W., Beckwith, S., \& Koelle, E. (2003). Advanced water distribution modeling and management 